\title{
A MEMS implementation of a Brownian Ratchet
}

\author{
Andrew Allison \& Derek Abbott \\ Centre for Biomedical Engineering (CBME) and \\ Department of Electrical and Electronic Engineering, \\ University of Adelaide, SA, 5005, Australia.
}

\begin{abstract}
A Brownian Ratchet is a device that can rectify the random Brownian motion of particles to yield a directed steady-state flow. We can imagine a thermo-fluid field of particles which interact with the ratchet. The laws of thermodynamics imply that the ratchet must use energy from some other source.

The dynamics of continuous-time Brownian ratchets are determined by a stochastic partial differential equation. We have used a simplified discrete-time model of a Brownian ratchet called "Parrondo's games" which are governed by a difference equation. In their original form, Parrondo's games are a finite set of simple games of chance. An indefinite pure sequence of any single game is neutral or even losing. A periodic or randomised sequence of mixed games can be winning. There is a steady state flow of probability in the preferred direction.

We have been able to design a feasible and consistent device, by mapping the conservation law of total probability onto the law of conservation of charge. This device can absorb energy from a mechanical field to produce a directed flow of charge. The fundamental architecture is based on a "bucket-brigade" device. The capacitors are 2-port MEMS devices. We use CMOS transmission gates to connect the capacitors in the required topology.

We present an analysis and simulation of the MEMS Brownian ratchet and suggest some possible applications.
\end{abstract}

Keywords: Brownian Ratchet, MEMS, re-parametrization

\section{BROWNIAN RATCHETS}

Brownian Motion was first observed by the Scottish botanist, Robert Brown, in the summer of 1827, following his voyage to Australia in 1822 . He noticed that grains of pollen were subject to perpetual random agitated movements when they were suspended in water. A typical sample path is shown in Figure 1. The phenomenon was studied

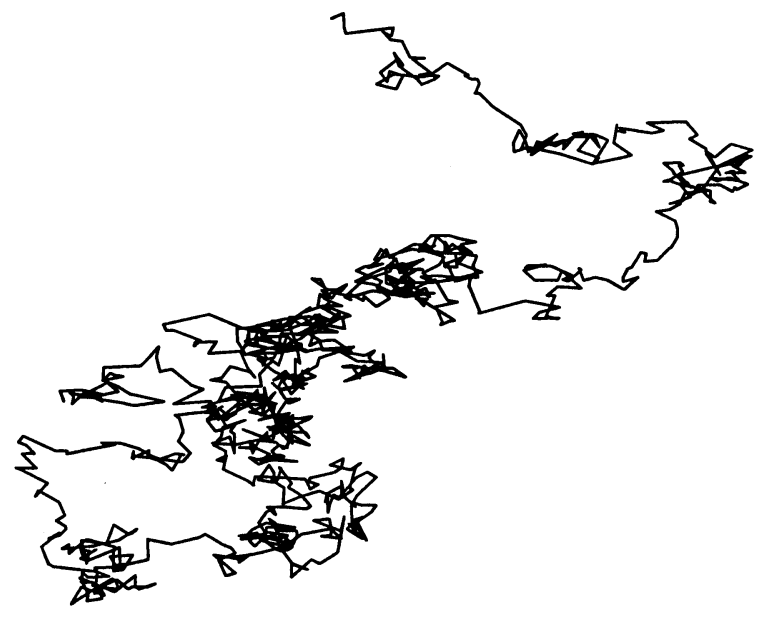

Figure 1. A sample path of a Brownian Motion.

Please send correspondence to Andrew Allison: E-mail: aallison@eleceng.adelaide.edu.au 
experimentally for the remainder of the 19th century and was not completely understood until the work of Marian Smoluchowski and and Albert Einstein. ${ }^{1}$

In 1871, James Clerk Maxwell published a thought experiment ${ }^{2}$ involving "a being whose faculties are so sharpened that he can follow every molecule in its course." Maxwell came to the shocking conclusion that such a being, now known as a "Maxwell's demon," might harness the microscopic movements of molecules to perform useful macroscopic work in a way that would violate the second law of Thermodynamics. Later works by Szilard, Brillouin, Landauer and Bennett have shown that the decisions made by the "demon" supply information (or neg-entropy) to the system and that this information cannot be supplied without continually re-setting the mental state of the demon. This entails an energy cost. Maxwell's demon is therefore really a heat engine. It is an unusual and exotic heat engine but it does not violate the second law.

In 1912 Marian Smoluchowski ${ }^{3}$ examined a similar thought experiment where the molecules interacted with the machinery through a paddle wheel and the selective role of the demon was taken by ratchet and pawl mechanism. Smoluchowski's ratchet appeared to harness the random Brownian motion of the paddle wheel in order to perform useful macroscopic work. Marian Smoluchowski, and later Richard Feynman, ${ }^{4}$ concluded that such a machine would work but that it would require a constant input of energy in order to prevent the pawl from reaching thermodynamic equilibrium with its surroundings. Smoluchowski's ratchet is also seen to be an unusual heat engine which does not violate the second law. A machine based on these principles is called a Brownian ratchet.

\subsection{Flashing ratchets}

There is quite an extensive literature on Brownian Ratchets ${ }^{5-11}$ and it is still a subject of active research. The contemporary model for a Brownian ratchet does not have the same physical appearance as the rotary "ratchet and pawl" machine of Smoluchowski. A modern paradigm is that of a "flashing ratchet" where an asymmetrical field is "flashed" on and off. ${ }^{9}$ This is illustrated in Figure 2, parts (a), (b) and (c).

(a)

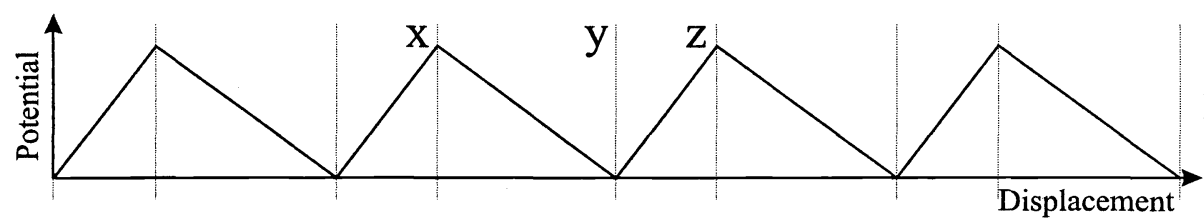

FIELD

(b)

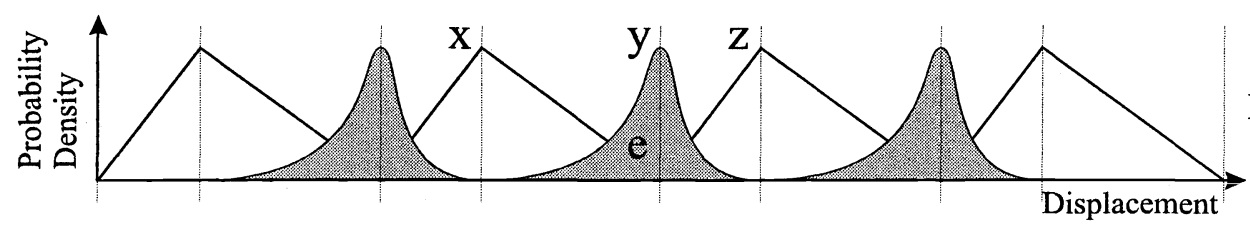

(c)

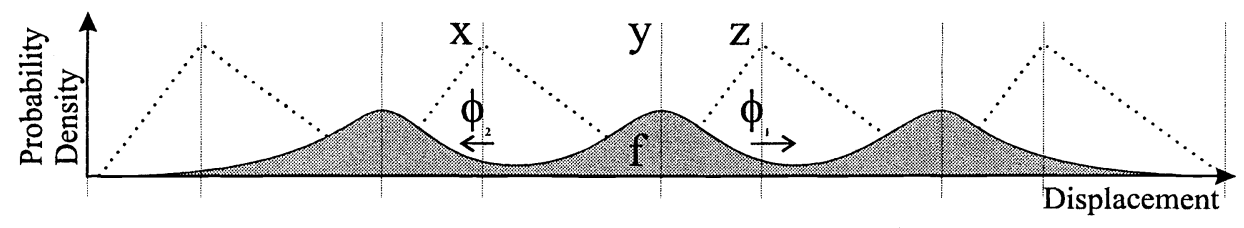

Figure 2. A Flashing ratchet.

In part (a) we see the asymmetrical form of a potential field. Potential is considered to be energy per unit material in the working substance. This could be Voltage (electrical potential) or it could be any potential associated with a conservative field. This potential is cycled "on" and "off" periodically. We imagine particles in the ratchet moving in response to the field and to gradients of particle concentration (or probability density).

In part (b) we see the steady-state distribution of particles in the field. They are confined most strongly to the regions of least potential, such as points near reference plane " $y$." We assume that the potential is strong enough to prevent significant barrier penetration through planes " $\mathrm{x}$ " and "z." The entire distribution of particles is divided into a number of sub-distributions. 


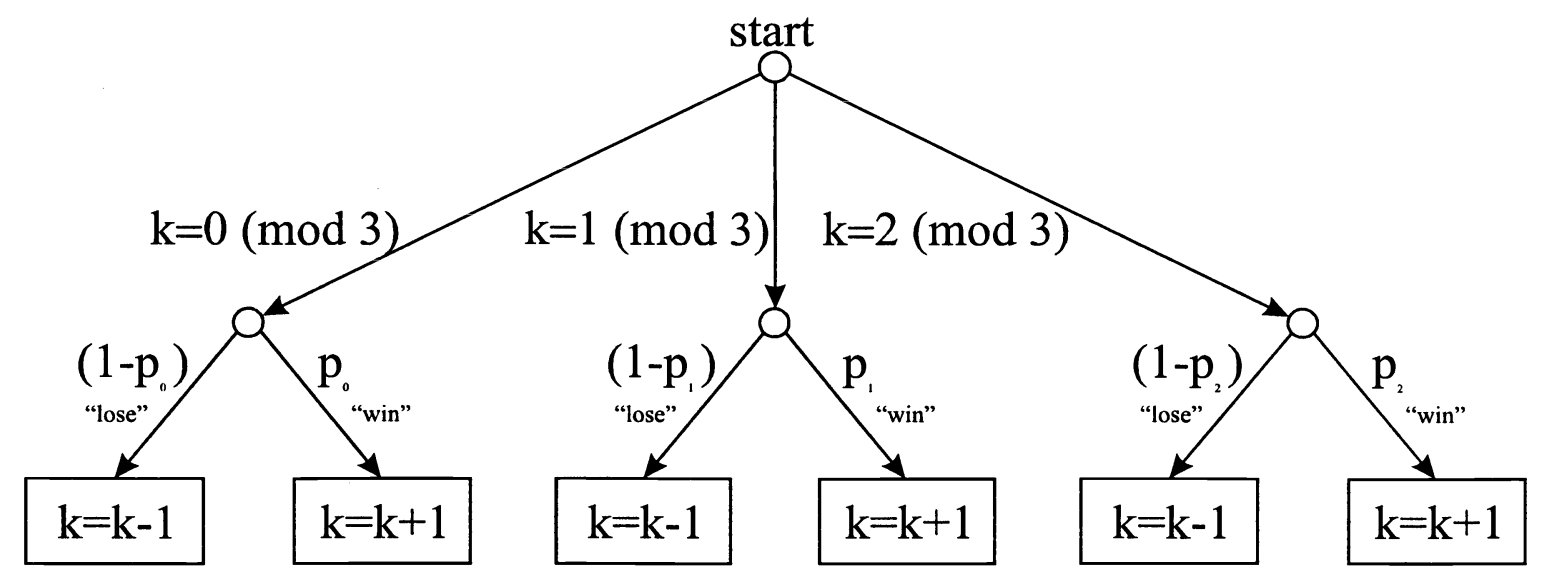

Figure 3. Structure of a single trial of a Parrondo's Game.

In part (c) we see the effect of removing the field. The non-steady-sate, non-equilibrium diffusion of particles causes the sub-distribution at " $y$ " to spread out. The concentration of particles at reference plane " $y$ " is reduced and there are fluxes of particles, $\Phi_{1}$ and $\Phi_{2}$, passing through reference planes " $\mathrm{z}$ " and " $\mathrm{x}$ " respectively. The forward flux, $\Phi_{1}$, is larger than the reverse flux, $\Phi_{2}$, because most of the particles were initially located closer to reference plane "z" than to reference plane " $\mathrm{x}$ " so $\Phi_{1}>\Phi_{2}$.

The effect over the entire ratchet is a superposition of the effects from each of the local sub-distributions. The precise rule for this combination is determined by a stochastic partial differential equation called the Fokker-Planck equation ${ }^{12-14}$ :

$$
\frac{\partial p}{\partial t}=-\frac{\partial(\alpha p)}{\partial y}+\frac{1}{2} \frac{\partial^{2}(\beta p)}{\partial y^{2}}
$$

where $p(y, t \mid x, t)$ is the transition probability density, $\alpha(x, t)$ is the infinitessimal first moment and $\beta(x, t)$ is the infinitessimal second moment. Equation 1 is also known as the Kolmogorov equation or the "master" equation. The functions $\alpha(x, t)$ and $\beta(x, t)$ are related to the potential function and to the laws of diffusion, such as Fick's law. The result of this ratchet process is that there is a net flow of particles to the right. This flow is paid for by the energy cost of asserting the field after diffusion has occurred.

When the field is re-asserted, particles that have crossed reference plane " $\mathrm{z}$ " are now pushed to the right and form part of the next sub-distribution. Particles that have crossed reference plane " $x$ " are pushed to the left and form part of the previous sub-distribution. The ratchet then settles down again towards the steady-state distribution shown in part (b).

Given the technical complexity of the Fokker-Planck equation, it is expedient to use a simplified model that captures the essential features of the original complex system. We can aggregate the probability densities of the various sub-distributions into single point probabilities, $V_{t, k}$, and we can aggregate the various fluxes between these sub-distributions into transition probabilities, $A_{i, j}$. We can aggregate each of the phases (b) and (c) Figure 2 into single time "ticks" of a discrete time system. The earliest known model of this type was proposed by Juan Parrondo. ${ }^{15}$

\section{PARRONDO'S DISCRETE TIME RATCHET}

Parrondo's games are a simplified discrete-time model of a flashing ratchet. ${ }^{15-18}$ They are formulated as a set of state-dependent games of chance. We could think of them as a generalised version of the Bernoulli trials where the coins are possibly biased and the coin that will be tossed next is determined by the present amount of capital, $k$. The process for these games can be represented by a decision tree which is shown in Figure 3 . The results of these decisions, and the subsequent trials, affect the state of the player, $k$. The Parrondo process is equivalent to the 


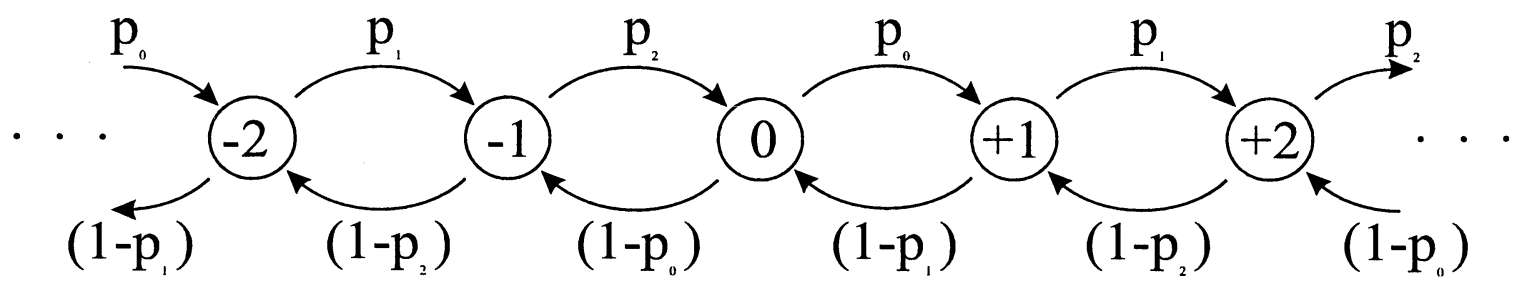

Figure 4. State transitions of a Parrondo's game.

operation of an indefinitely large non-deterministic state machine. Part of this machine is represented in in Figure 4. In Parrondo's games, the state-variable, the capital, $k$ and the spatial "displacement" are considered to be equivalent. The asymmetrical potential function is represented indirectly through the state dependent probabilities $\left[p_{0}, p_{1}, p_{2}\right]$. Exact information about potential and energy has been lost in the transformation to discrete time. The (Newtonian) law of conservation of matter has been replaced by the law of total probability. The probability functions shown in Figure 2 are replaced by a time dependent probability vector :

$$
\underline{V_{t}}=\left[\cdots, V_{t,-2}, V_{t,-1}, V_{t, 0}, V_{t,+1}, V_{t,+2}, \cdots\right] .
$$

It can be shown that the time evolution of Parrondo's games can be represented as a non-homogeneous Markov chain. We can represent the time evolution of the system using simple algebraic notation. For the effect of one trial of "Game A" we could write:

$$
\underline{V_{(t+1)}}=\underline{V_{t}} \cdot\left[A_{i, j}\right] \text {. }
$$

For a single play of "Game B" we could also write:

$$
\underline{V_{(t+1)}}=\underline{V_{t}} \cdot\left[B_{i, j}\right] .
$$

It is understood that games " $\mathrm{A}$ " and " $\mathrm{B}$ " and possibly other games could be included in the sequence. In general the sequence is mixed, with different games being played at different times. The matrices all have the form :

$$
\left[A_{i, j}\right]=\left[\begin{array}{cccccccccc}
\ddots & \ddots & & & & & & & & \\
\ddots & 0 & a_{0} & & & & & & & \\
& \left(1-a_{1}\right) & 0 & a_{1} & & & & & & \\
& & \left(1-a_{2}\right) & 0 & a_{2} & & & & & \\
& & & \left(1-a_{0}\right) & 0 & a_{0} & & & & \\
& & & & \left(1-a_{1}\right) & 0 & a_{1} & & & \\
& & & & \left(1-a_{2}\right) & 0 & a_{2} & & \\
& & & & & \left(1-a_{0}\right) & 0 & a_{0} & \\
& & & & & & & \left(1-a_{1}\right) & 0 & \ddots \\
& & & & & & & \ddots & \ddots
\end{array}\right]
$$

All of the elements which are not explicitly represented are zero. The variation along the diagonals has a period of 3. Parrondo's original specification for the games was:

Game "A" : $\left[a_{0}, a_{1}, a_{2}\right]=\left[\frac{99}{200}, \frac{99}{200}, \frac{99}{200}\right]$

Game "B": $\left[b_{0}, b_{1}, b_{2}\right]=\left[\frac{19}{200}, \frac{149}{200}, \frac{149}{200}\right]$.

Game "A" corresponds approximately to the process in Figure 2 (c) and game "B" corresponds approximately to the process in Figure $2(\mathrm{~b})$.

The natural way to measure flow within Parrondo's games is to estimate asymptotic rates of increase in the expected value of the capital, $k$ as a function of time. Simulations of Parrondo's original games yield a rate of return 
$Y \approx+0.0166$. That is, for every dollar that we invest we expect to make a long term profit of $\$ 0.0166$ per time tick. The rate of flow is modest but positive, in contrast to the pure sequences which are negative.

The fact that Parrondo's games use discrete-time means that they could be implemented within a clocked architecture using silicon, as long as we could find a rigorous way to re-parametrize the equations.

\section{ASYMPTOTIC RATE OF TRANSPORT FROM PARRONDO'S RATCHET}

If we were designing a ratchet, or a set of games, then we might have some free choice of the conditional probabilities, $\left[a_{0}, a_{1}, a_{2}\right]$ and $\left[b_{0}, b_{1}, b_{2}\right]$, and we would need to know how the rate of return, $Y$, depended on these conditional probabilities. It is possible to apply the theory of Markov chains with rewards ${ }^{19}$ to this problem and it can be shown that:

$$
Y=Y_{0} \cdot\left(\frac{1-\Gamma}{1+\Gamma}\right)
$$

where $Y_{0}=3$ and $\Gamma$ is given by

$$
\Gamma=\frac{1+\left(\left(1-a_{0}\right)\left(1-a_{1}\right)\left(1-a_{2}\right)\right)}{1+\left(a_{0} a_{1} a_{2}\right)} .
$$

Equation 5 only strictly applies to a homogeneous sequence of games "A" but these formulae can be applied to the more general mixed case by applying the formulae in the appropriate sequence. There must be a rule to specify the sequence of games, $\{A, B, B, A, B, \cdots\}$, and this must be taken into account when calculating the asymptotic rate of return.

\section{OPTIMUM FORM OF THE RATCHET}

Simulations reveal that periodic sequences yield the greatest return. Further investigation by the authors, using Genetic Algorithms, reveal that the most effective form of the games is a set of 3 games that are played in a strict periodic sequence $\left\{G_{0}, G_{1}, G_{2}, G_{0}, G_{1}, G_{2}, \cdots\right\}$. The transition probabilities are as follows :

Game $G_{0}: \quad[\mu,(1-\mu),(1-\mu)]$

Game $G_{1}: \quad[(1-\mu), \mu,(1-\mu)]$

Game $G_{2}: \quad[(1-\mu),(1-\mu), \mu]$

where $\mu$ is a very small probability, $0<\mu<1$. We can think of $\mu$ as being a very small, ideally "microscopic", positive number. The games are most effective as $\mu \rightarrow 0$ where the return is $Y \rightarrow 1$. Each game has two conditional probabilities that are close to 1.0 and are almost certainly winning. Each game has one conditional probabilities that is close to 0.0 and is almost certainly losing. The losing part of the game represents a barrier. The reason for including the parameter, $\mu \neq 0$, is that the ideal case, $\mu=0$, is not feasible in practice. We must be content with an approximation. The rate of return form any pure sequence of these games is approximately

$$
Y \approx \frac{1}{2} \mu
$$

which is very close to zero and yet the return from the cyclic combination of these games is approximately

$$
Y \approx 1-3 \mu
$$

which is very close to a certain win. We can engineer a situation where we achieve an almost certain win every time out of games that, on their own, deliver almost no benefit at all. These games work better as a team than on their own. 


\section{RE-PARAMETRIZATION OF EQUATIONS FOR THE RATCHET}

If we examine Figure 4 or consider a single row from Equations 2 or 3 then we can write

$$
\left.\left.V_{(t+1), n}=V_{t,(n-1)} \cdot p_{t,((n-1)} \quad(\bmod 3)\right)+V_{t,(n+1)} \cdot p_{t,((n+1)}(\bmod 3)\right)
$$

where $p_{t, k}$ are the particular conditional transition probabilities that apply at time $t+1$. The conserved quantity (probability) at time $t+1$ is a weighted sum of the neighbouring conserved quantities at an earlier time $t$. If we wish to map the law of conservation of charge onto the law of total probability then we need a discrete mechanism for creating weighted sums of charge. One very simple mechanism for achieving this is to use the (normally undesirable) effect of charge sharing. ${ }^{20,21}$

\subsection{Probabilistic interpretation of charge sharing}

In Figure 5 we see two capacitors separated by a CMOS transmission gate ${ }^{22}$ which is driven by complementary clock signals, $\Phi$ and $\bar{\Phi}$. Suppose that $\Phi$ is initially low and $C_{1}$ and $C_{2}$ are at initial voltages $V_{1}$ and $V_{2}$ respectively. The

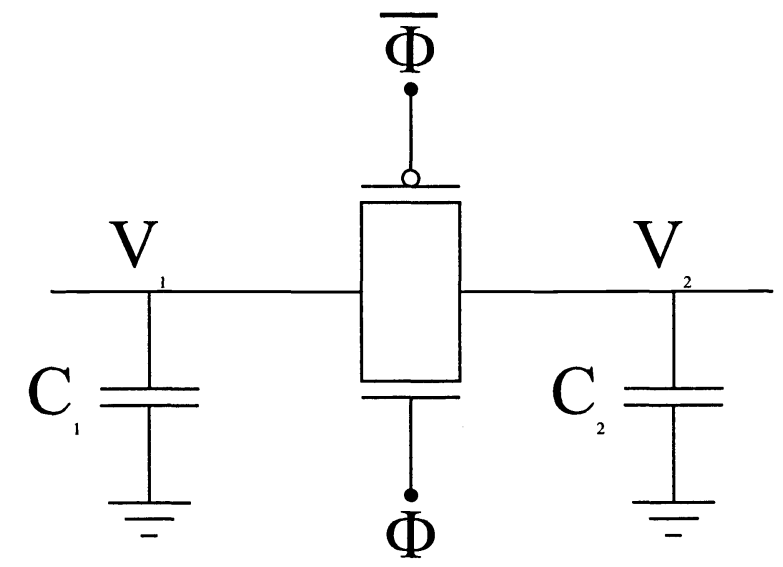

Figure 5. A transmission gate structure with charge sharing

stored charges in $C_{1}$ and $C_{2}$ are given by

$$
Q_{1}=C_{1} V_{1} \Leftrightarrow V_{1}=\frac{Q_{1}}{C_{1}}
$$

and

$$
Q_{2}=C_{2} V_{2} \Leftrightarrow V_{2}=\frac{Q_{2}}{C_{2}} .
$$

The transmission gate is now closed as $\Phi$ goes high. We assume that the time constant of the resulting circuit is small compared with the period of the clock. Both capacitors now move to an equilibrium distribution of charge and a new voltage $V_{3}$. Equations 10 and 11 together with the conservation of charge now imply that

$$
V_{3}=\left(\frac{C_{1}}{C_{1}+C_{2}}\right) \cdot V_{1}+\left(\frac{C_{2}}{C_{1}+C_{2}}\right) \cdot V_{2} .
$$

The equations for charge sharing now become

$$
Q_{1}^{\prime}=\left(\frac{C_{1}}{C_{1}+C_{2}}\right) \cdot\left(Q_{1}+Q_{2}\right)
$$

and

$$
Q_{2} \prime=\left(\frac{C_{2}}{C_{1}+C_{2}}\right) \cdot\left(Q_{1}+Q_{2}\right)
$$


where $Q_{1}$ ' and $Q_{2}$ ' are the new amounts of charge stored in the capacitors. This is consistent with conservation of charge and $\left(Q_{1} \prime+Q_{2} \prime\right)=\left(Q_{1}+Q_{2}\right)$. If we think of the charge as being like an "electron gas" that occupies the two capacitors then the fractions $\mu=C_{2} /\left(C_{1}+C_{2}\right)$ and $(1-\mu)=C_{1} /\left(C_{1}+C_{2}\right)$ are the probabilities that any given electron will be found in one or other of the two capacitors. Probability and charge map linearly onto one another. The scaling factor is simply the number of unit electron charges in the system. We know, from Equations 7 and 8 that we would like $\mu$ to be very close to 0 , or possibly to 1 , but this is not completely feasible since $C_{1}$ and $C_{2}$ must be finite positive numbers. The formal expressions for $\mu$ are:

$$
\mu=\left(\frac{Q_{2}^{\prime}}{Q_{1^{\prime}}+Q_{2} \prime}\right)=\left(\frac{C_{2}}{C_{1}+C_{2}}\right)
$$

and

$$
(1-\mu)=\left(\frac{Q_{1}^{\prime}}{Q_{1}^{\prime}+Q_{2} \prime}\right)=\left(\frac{C_{1}}{C_{1}+C_{2}}\right)
$$

\subsection{MEMS variable capacitors}

If the capacitors could not vary then every cycle of this machine would contribute to charge sharing in such a way that all capacitors would tend towards the same potential. This is shown in the simulation in Figures 8 and 9 . This is analogous to Parrondo's games where we toss a completely fair coin, or to unconstrained diffusion. We can not achieve the ratcheting effect without the ability to vary the conditional probabilities in Equation 9 or the proportion of charge sharing in Equations 13 and 14. This means that we must be able to vary $C_{1}$ and $C_{2}$ and every other capacitor in the ratchet. This will requite mechanical work that must be provided by an actuator. We propose the use of comb drive actuators ${ }^{23}$ as both variable capacitors and actuators. These should be arranged in pairs and be connected mechanically but remain electrically isolated. A schematic diagram of a comb drive actuator is shown in Figure 6. One actuator can be driven to vary the capacitance of the other. The pair of actuators can be regarded,

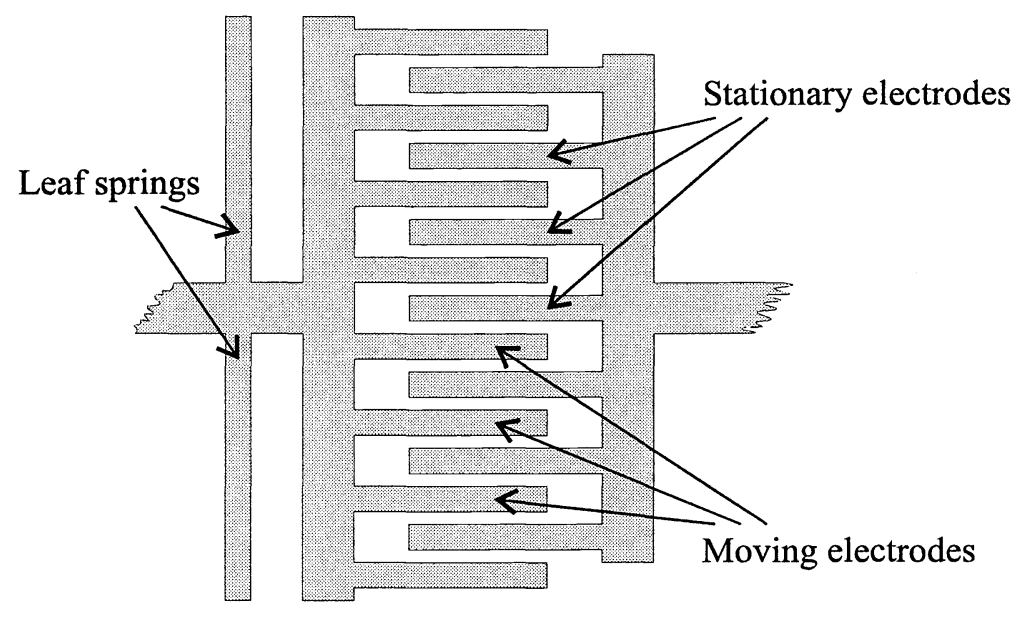

Figure 6. Comb drive actuator

from a mechatronic point of view, as an electrical 2-port device. This lends itself to analysis using the method of bond-graphs. ${ }^{24}$ We can think of a pair of "back to back" comb drives as an energy-storing transducers.

If we allow $C_{1}$ and $C_{2}$ to vary then we can think of the ratio of change of $\mu$, ie: $\mu_{t 2} / \mu_{t 1}$, as being like a compression ratio in mechanics. It is very difficult to make this large because of leakage in the storage elements or breakdown of the switching elements.

\section{A FOUR STROKE SEQUENCE OF OPERATION}

In Figure 2 we consider the ratchet to have two phases or "strokes." We can use the word "stroke" in reference to classical thermodynamic cycles such as the Carnot, Diesel, Otto and Stirling cycles. The word "phase" is a reference 
to non-overlapping clock phases in a clocked digital circuit. In the MEMS ratchet, the concepts of "stroke" and "phase" converge.

The laws of Parrondo's games require that the probability of remaining in the same state for two successive time ticks is zero. This is clear from the graphical definitions in Figures 3 and 4 . The problem is that if we use the charge sharing method, described in Equations 13 and 14, then we can not entirely exclude all charge from a capacitor, no matter how forceful our actuator may be. The easiest way around this it to simply switch the capacitor electonically to some other location. When we perform our accountancy to check for conservation of charge we will not find any charge at that location. Some of the charge can then be switched back to the original location in the next part of the cycle. We can avoid the use of a large amount of complicated controlling circuitry if we settle for two asymmetrical cycles of two phases rather than one symmetrical cycle of two phases. This is indicated in Figure 7 . The sequence in time is a cyclic repetition of $\{(a),(b),(c),(d)\}$. Phases (a) and (c) are diffusion phases where the carriers spread out to an equilibrium distribution. These are analogous to the process in Figure 2 (c). Phases (b) and (d) are compression phases where the capacitors are changed in value because they are driven by the comb actuators. Charge is actively "compressed" into one or other of the capacitors in accordance with Equations 13 and 14. These compression phases are analogous to the process in 2 (b).

The aim is always to move as much charge as possible to the right, in the "positive" direction. This means that in Figure 7 (b) we need to increase $C_{2}$ and $C_{4}$ and decrease $C_{1}$ and $C_{3}$. In Figure. 7 (d) we need to decrease $C_{2}$ and $C_{4}$ and increase $C_{1}$ and $C_{3}$. These should be done using the maximum possible compression ratio. It should be noted that the voltages $V_{1}$ to $V_{4}$ vary with time.

\section{SIMULATION OF THE ELECTRICAL CIRCUIT}

We tested the electrical part of the system using P-SPICE. The results are shown in Figures 8 and 9 . We have used a split power supply to allow signals of either sign. This is not a necessary feature for a MEMS ratchet. The source $V_{0}$ is connected to capacitor $C_{1}$ through a diode. This to allow the initial charge of $C_{1}$. No further charge is injected onto the system after this. The simulation shows the effect of charge sharing. The charge from $C_{1}$ gradually propagates down the ratchet, as the transmission gates are switched. This is quite analogous to the diffusion of charge in a conductive dielectric, which is governed by Poisson's equation.

As charge is shared, all the capacitors acquire the same voltage. In order to investigate the charge pumping effect of the ratchet, we would have to write our own simulator in a mathematical language, such as Matlab. This is clearly the next stage of the work.

\section{ANALOGIES TO OTHER MACHINES}

Machines that use mechanical energy to change the value of a capacitive system to generate large potentials are not new. The classical examples are Volta's Electrophorus and the Wimshurst machine. The idea of using MEMS technology to implement ratchets has already been proposed by Abbott et. al.. ${ }^{25}$ A MEMS electrostatic ratchet could be a method of generating large potentials in a small space on a chip.

In their optimal form, the action of Parrondo's games is very much like a peristaltic pump or a stepper motor. The system is driven in a tightly controlled manner from one state to the next. The emphasis is on accurate control or measure. The peristaltic pump can deliver an accurate amount of a substance at a required moment in time. The stepper motor can produce an accurate rotation or translation. The MEMS ratchet could be used to accurately deliver a known amount of charge at a specified time. This could have application in instrumentation.

\section{SUMMARY}

We have indicated how Parrondo's games could be re-parametrized in a form that could be constructed using MEMS technology. The main aim of this work is to carry out a "thought experiment" to bring physical principles, such as energy, back into the study of discrete time ratchets. There are also some possible applications

- To induce large electrical potentials

- To deliver controlled amounts of charge

although more detailed analysis and simulation are required. 

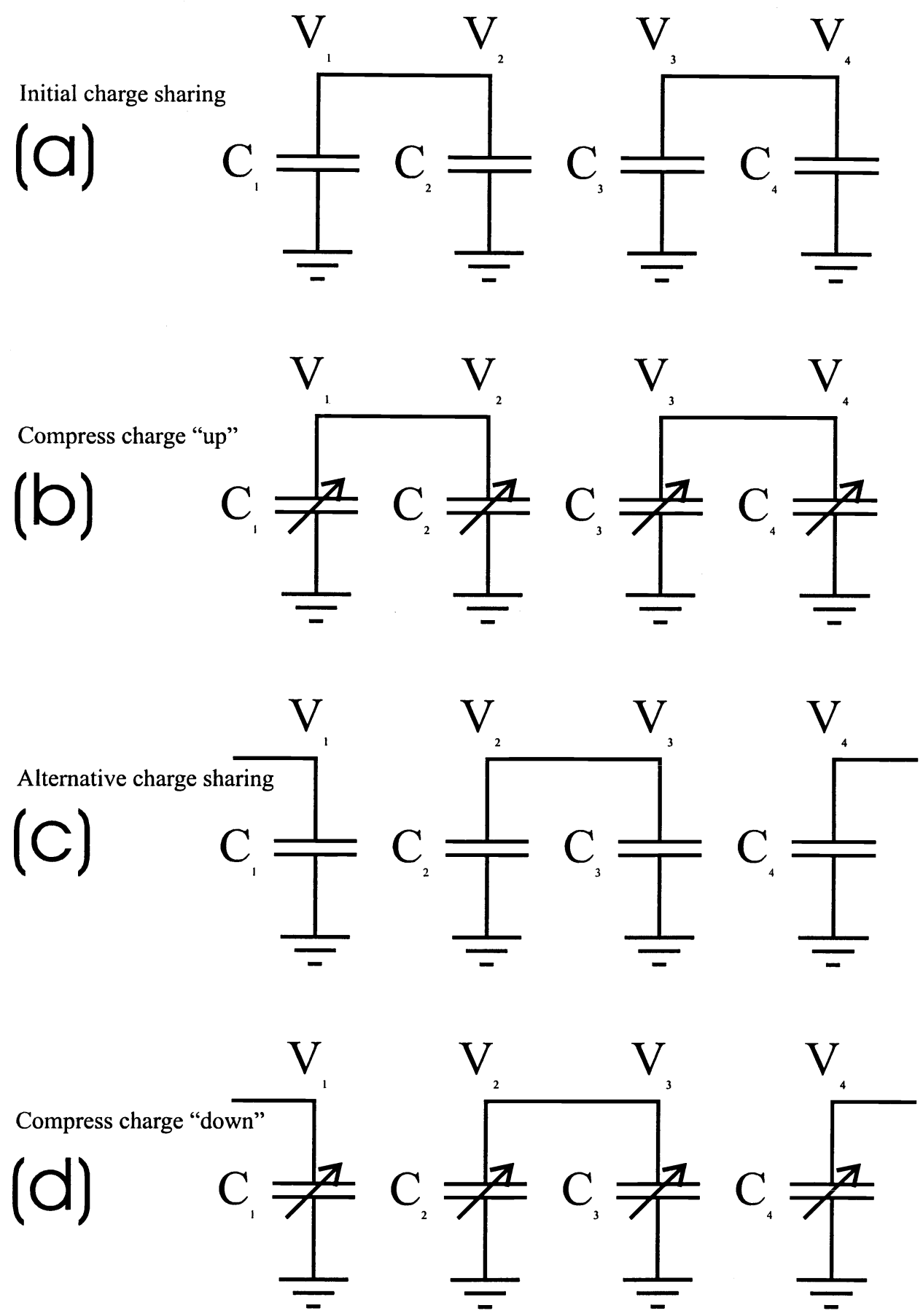

Figure 7. A four stroke plan for swapping charge between capacitors

\section{REFERENCES}

1. A. Einstein, "Investigations on the theory of the brownian movement," Ann. Physik. 17(17), 1905.

2. J. C. Maxwell, Theory of heat, D.Appleton and co., New York, 1871.

3. M. Smoluchowski, "Experimentall nachweisbare, der ulblichen thermodynamic widersprechende moickularphanomene," Phys. Z. 13(13), pp. 1069-1080, 1912. 


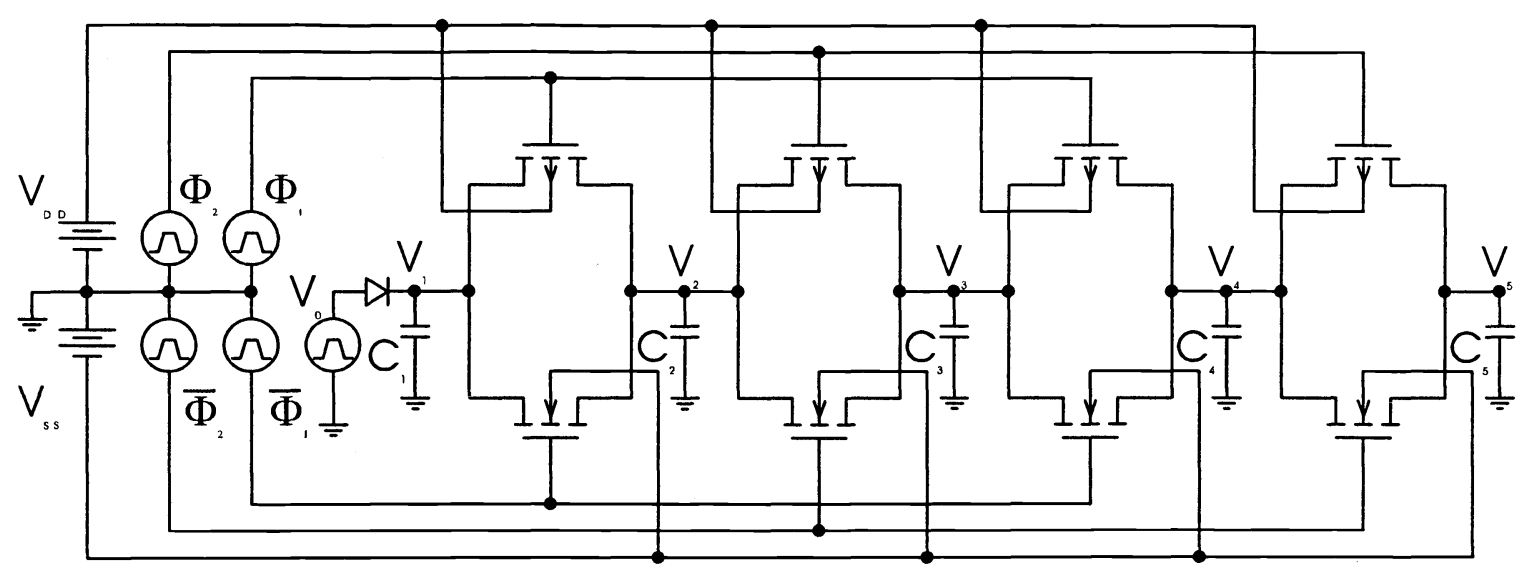

Figure 8. SPICE model for the transmission gates

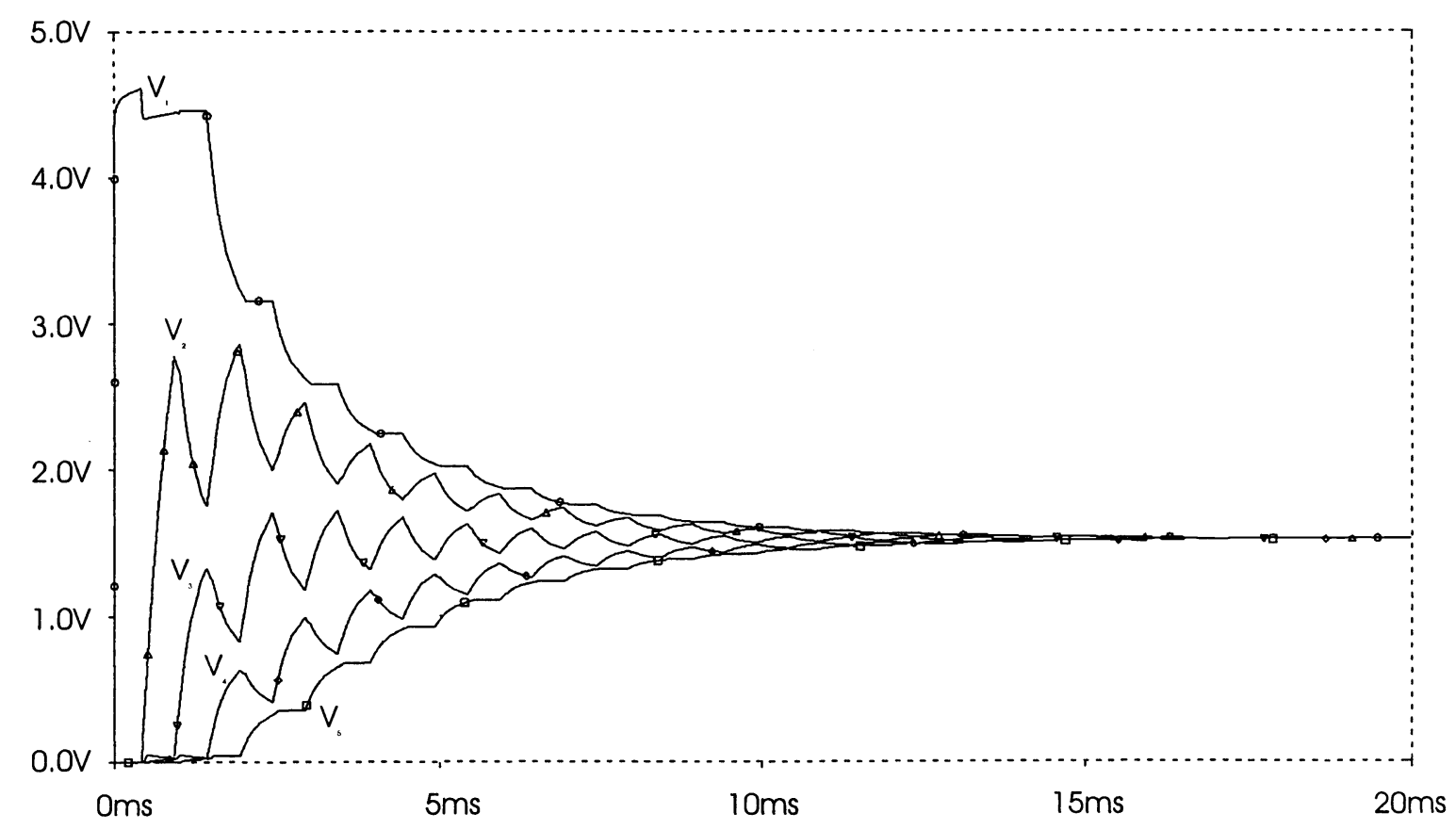

Figure 9. Result from SPICE simulation, showing charge sharing.

4. R. P. Feynman, R. B. Leighton, and M. Sands, The Feynman Lectures on Physics, vol. 1, Addison-Wesley, Reading, MA, 1963.

5. R. D. Astumian, P. B. Chock, T. Y. Tsong, Y. Chen, and H. V. Westerhoff, "Can free energy be transduced from electric noise?," Proc. Natl. Acad. Sci. USA 84, pp. 434-438, 1987.

6. R. D. Astumian and M. Bier, "Fluctuation driven ratchets: Molecular motors," Physical Review Letters 72 , pp. 1766-1769, 1994.

7. J. Rousselet, L. Salome, A. Ajdari, and J. Prost, "Directional motion of brownian particles induced by a periodic asymmetric potential," Nature 370, pp. 446-448, 1994.

8. C. R. Doering, W. Horsthemke, and J. Riordan, "Nonequilibrium fluctuation-induced transport," Physical Review Letters 72, pp. 2984-2987, 1994. 
9. C. R. Doering, "Randomly rattled ratchets," Nuovo Cimento 17D, pp. 685-697, 1995.

10. M. Bier, "Reversals of noise induced flow," Physics Letters A A211, pp. 12-18, 1996.

11. M. Bier, "Brownian ratchets in physics and biology," Contemporary Physics 38, pp. 371-379, 1997.

12. A. Papoulis, Probability, Random Variables and Stochastic Processes, McGraw-Hill Inc, 1991.

13. F. Reif, Statistical and Thermal Physics, McGraw Hill, 1985.

14. H. C. Tuckwell, Elementary Applications of Probability Theory, Chapman \& Hall, 1995.

15. G. P. Harmer, D. Abbott, P. G. Taylor, and J. M. R. Parrondo, "Parrondo's paradoxical games and the discrete brownian ratchet," in Abbott, ${ }^{26}$ pp. $149-160$.

16. G. P. Harmer, "Parrondo's paradox," Statistical Science 14, pp. 206-213, 1999.

17. C. E. M. Pearce, "On parrondo's paradoxical games," in Abbott, ${ }^{26}$ pp. $420-425$.

18. C. E. M. Pearce, "Entropy, markov information sources and parrondo games," in Abbott, ${ }^{26}$ pp. $426-431$.

19. R. A. Howard, Dynamic Programming and Markov Processes, John Wiley \& Sons Inc. , 1960.

20. J. C. Chang and B. J. Sheu, The Circuits and Filters Handbook, ch. 63.3, pp. 1993-1997. CRC Press and IEEE Press, 1995.

21. M. Shoji, Theory of CMOS Digital Circuits and Circuit Failures, Princeton University Press, 1992.

22. R. C. Chang and B. J. Sheu, The Circuits and Filters Handbook, ch. 62.1, pp. 1903-1912. CRC Press and IEEE Press, 1995.

23. W. C. Tang, T. Cuong, H. Nguyen, and R. T. Howe, "Laterally driven polysilicon resonant microstructures," Proceedings IEEE Micro Electro Mechanical Systems 9, pp. 53-59, February 1989.

24. D. C. Karnopp, D. L. Margolis, and R. C. Rosenberg, System Dynamics : Modeling and Simulation of Mechatronic Systems, John Wiley \& Sons Inc., New York, 2000.

25. D. Abbott, B. R. Davis, and J. M. R. Parrondo, "Detailed balance of the feynman micromotor," Proceedings of the SPIE 3891, pp. 184-189, 1999.

26. D. Abbott, ed., Unsolved Problems of Noise and Fluctuations '99, 1999. 